\title{
Preface: Biomass Conversion Over Heterogeneous Catalysts: Contributions from the 2011 AIChE Annual Meeting
}

\author{
Carsten Sievers
}

Published online: 27 March 2012

(C) Springer Science+Business Media, LLC 2012

The conversion of biorenewable feedstocks to fuels and chemicals has received tremendous interest over the last years. This interest is being fueled by concerns regarding depleting oil reserves and the connections of carbon dioxide emission with climate change. In addition, energy independence has become a political goal of several countries. Although biofuels have been produced commercially in Brazil for over 25 years there is an enormous need for research and development. For example, differences in composition of biomass feedstocks and their availability in different geographic locations will require the development of specific novel processes.

Catalysts play a critical role in the vast majority of processes in the chemical and petrochemical industry. Process economic are inextricably linked to the savings in energy and reduction of waste that these catalysts provide. In particular, the use of heterogeneous catalysts is attractive because they are readily removed from reaction mixtures so that they can be reused. Although tax incentives can help during the startup stage, future biorefineries will have to reach a level of efficiency that allows them to compete with established industrial plants. It is hard to imagine that this will be possible without the use of efficient and affordable catalysts that have high activity and selectivity as well as a long lifetime. Significant research efforts will be required to develop catalysts that meet all of these requirements. Fortunately, numerous research groups are addressing this challenge by developing novel catalysts and catalytic processes.

The promise of heterogeneously catalyzed processes for biomass conversion and the technological challenges of the development of suitable catalysts for these processes were reflected by the enormous number of related contributions at the 2011 AIChE Annual Meeting in Minneapolis. Dedicated sessions on catalytic biomass conversion were organized by the Catalysis and Reaction Engineering Division and the Sustainable Engineering Forum. In addition, numerous contributions in other sessions addressed related topics. This shows that the chemical engineering community has identified the development of sustainable processes based on biorenewable resources as one of the critical challenges of the twentyfirst century.

The manuscripts of this special issue of Topics in Catalysis represent the breadth of the papers at the 2011 AIChE Annual Meeting. Hydrotreating and cracking of biomass derived oxygenates are discussed in multiple contributions, and several manuscripts present work on processing biomass feedstocks in aqueous phase over acid and/or metal catalysts. Additional topics are catalytic pyrolysis and reforming of tars in syngas.
C. Sievers $(\square)$

School of Chemical \& Biomolecular Engineering, Georgia

Institute of Technology, 311 Ferst Dr, Atlanta,

GA 30332-0100, USA

e-mail: carsten.sievers@chbe.gatech.edu 